\title{
Diagnostic 4.0 du système physique de production : modèle de référence d'audit des gammes de production
}

\author{
David Damand (iD) 1 , Marc Barth (D)2, Abdellatif Dkhil ${ }^{3}$ \\ ${ }^{1}$ HuManis laboratory of EM Strasbourg Business School, 61 avenue de la Forêt Noire, F-67000, Strasbourg, \\ France,damand@unistra.fr, \\ ${ }^{2}$ HuManis laboratory of EM Strasbourg Business School, 61 avenue de la Forêt Noire, F-67000, Strasbourg, \\ France, marc.barth@insa-strasbourg.fr, \\ ${ }^{3}$ Université du Québec, Ecole de Technologie Supérieure, LIPPS, 1100 rue Notre Dame Ouest, Montréal, H3C \\ 1K3, Canada, abdellatif.dkhil@gmail.com
}

\section{Résumé}

L'industrie 4.0 est le résultat de la convergence entre l'industrie et le numérique. L'industrie 4.0 repose sur une relation homme-machine plus collaborative. Cette collaboration de type réseau est caractérisée par une communication continue et instantanée entre les moyens de productions et d'approvisionnement. Ce qui permet une surveillance continue d'éventuelles dérives de performance du système physique de production. Cependant, la qualité de cette nouvelle organisation en réseau des moyens de production dépend de la qualité des conditions initiales du système physique de production. Pour contribuer au diagnostic du système physique de production, ce papier propose un modèle de référence d'audit des gammes de production.

Mots clés : audit et diagnostic, propriétés d'analyse, système physique de production, visualisation des connaissances

\section{Reference model for the analysis of physical production system data for a diagnosis 4.0}

\begin{abstract}
Industry 4.0 is the result of the convergence between industry and digital technology. Industry 4.0 is based on a more collaborative man-machine relationship. This network collaboration is characterized by a continuous and instantaneous communication between the means of production and supply. This allows for continuous monitoring of any deviations in performance of the physical production system. However, the quality of this new networked organization of production means depends on the quality of the initial conditions of the physical production system. In order to contribute to the diagnosis of the physical production system, this paper proposes a reference model for auditing production routings.
\end{abstract}

Keywords : audit and diagnosis, analysis properties, physical production system, knowledge visualisation 


\section{INTRODUCTION}

Un des défis de la $4 \mathrm{e}$ révolution industrielle intitulés " industrie 4.0 » est de répondre à cette exigence de produit unique et personnalisé tout en conservant des coûts équivalents à une production de masse et cela, malgré les faibles volumes de production engendrés. La réponse 4.0 se traduit en autres, par la connexion des besoins du client au système physique de production via l'évolution des systèmes de décision et d'information vers des systèmes cyber-physiques. Il est généralement admis que la performance de ces systèmes évolués de décision et d'information est dépendante de la performance du système physique de production (SPP) (Koren et al., 1998 ; Rouse et al., 2003). Compte tenu des enjeux financiers (Mayr et al., 2018), il faut pouvoir régulièrement diagnostiquer et le cas échéant reconcevoir le SPP.

Un SPP efficient, dit "Lean ", permettra de concevoir un système de décision et d'information efficient (Chanegrih \& Creusier, 2015, AmoakoGyampah et al, 2008 ; Singh \& Sharma, 2006). A contrario, la non-performance d'un SPP, dit « nonLean " est difficilement compensable par la performance du système de décision et d'information (Nicoletti, B. 2013 ; Olhager, 2013, Lyonnet \& Messaoudene, 2012, Real, R. et al., 2010).

La littérature scientifique dénombre depuis plusieurs décennies un nombre important de travaux sur la conception de SPP. Ces travaux proposent une décomposition séquentielle systématique du processus de conception du SPP en quatre phases (Irani et al., 2000 ; Muther, 1973 ; Phillips, 1997 ; Raoot et al., 1993 ; Singh \& Sharma, 2006 ; Tompkins et al, 2003) :

- Phase 1 : Audit et diagnostic de l'implantation du SPP,

- Phase 2 : Elaboration des agencements conceptuels du SPP,

- Phase 3 : Elaboration des agencements physiques du SPP,

- Phase 4 : Implémentation du SPP.
Les travaux développés dans ce papier se positionnent au niveau de la Phase 1. Celle-ci consiste à préciser la prise en compte ou la non-prise en compte des principes organisationnels du lean manufacturing dans l'implantation actuelle. Cette phase va permettre d'identifier les causes principales de pertes de performance ainsi que des principes de solutions potentielles.

Généralement différents principes de solution d'implantation potentielles sont analysés. La difficulté majeure est essentiellement liée au nombre important de points de vue à considérer : sécurité, formation, mouvement, transport, qualité produit, procédé, temps de production, gammes de production, etc. Ces points de vue sont en grande partie liés aux flux physiques des produits. Le flux physique des produits représente l'élément fondateur du SPP et est étroitement lié à la performance du SPP (Alfnes \& Martinsen, 2006 ; Leone et al., 2002 ; Tompkins et al., 2003). Le point de vue considéré dans ce papier est le point de vue du flux physique des produits modélisé dans les gammes de production, c'est-à-dire les déplacements des produits entre postes de charge, appelé par la suite : 'le flux des produits'.

Le type de gammes de production considérée dans ce papier sont des gammes industrielles de production : multiproduits (plusieurs centaines), multi-opérations (plusieurs milliers), multipostes de charge (plusieurs dizaines) sans prise en compte de gammes alternatives. Aussi, les relations entre postes de charge créées par le flux des produits sont potentiellement complexes. Cela renvoie à la question de l'intelligibilité du flux des produits.

Comprendre le flux des produits consiste à auditer les gammes de production pour en extraire les propriétés. Par exemple, les éléments de réponse aux quatre questions ci-après représentent des propriétés des gammes à auditer :

1. Est-ce que certains produits sont fabriqués suivant la même séquence d'opérations ?

2. Est-ce que certains produits sont fabriqués sur les mêmes postes de charge? 
3. Y a-t-il des postes de charge qui ont un nombre important d'échanges avec les autres postes de charge ?

4. Y a-t-il des postes de charge qui sont systématiquement utilisés en début ou en fin de gamme?

Si par exemple la réponse associée à la question 1 est " oui ", alors le flux des produits défini sur l'ensemble des postes de charge suit une structure d'ordre. Dans ce cas, le principe de solution à analyser lors du diagnostic de l'implantation correspond à une implantation de type "Ligne ». Le principe technologique de mise en œuvre revient à ordonner spatialement les postes selon l'ordre d'utilisation des postes de charge dans les gammes.

La question de recherche posée dans ce papier concerne la possibilité de créer un modèle de référence pour l'audit de gammes de production complexe dans un contexte dit « lean».

La suite du papier est structurée comme suit.

La section 3 décrit la méthode de construction du modèle de référence.

La section 4 décrit les règles d'instanciation du modèle de référence.
La section 5 décrit la validation du modèle de référence.

La section 6 conclut ce papier.

\section{REVUE DE LA LITTERATURE}

Le processus d'audit des gammes et de diagnostic de l'implantation est usuellement décomposé en 4 activités dans la littérature scientifique et représenté par un actigramme IDEFO (Figure 1).

Le processus d'audit des gammes de production (A1, $A 2, A 3)$ est décrit à l'aide de la taxonomie adaptée de Card et al. (1999) et Chi (2000). Par souci de précision, un cas d'école illustre la description de la phase 1 (Activité A1 à A4).

\section{A1. Transformation des données}

La transformation des données reformate les gammes de production (Tableau 1 ) en format plus adapté appelé table de données (Tableau 2). Les 2 tables de données usuelles sont : matrice (produits postes de charge), matrice (postes de charge postes de charge) orientée ou non. Ces tables de données représentent les informations à visualiser.

--- Audit des gammes de production

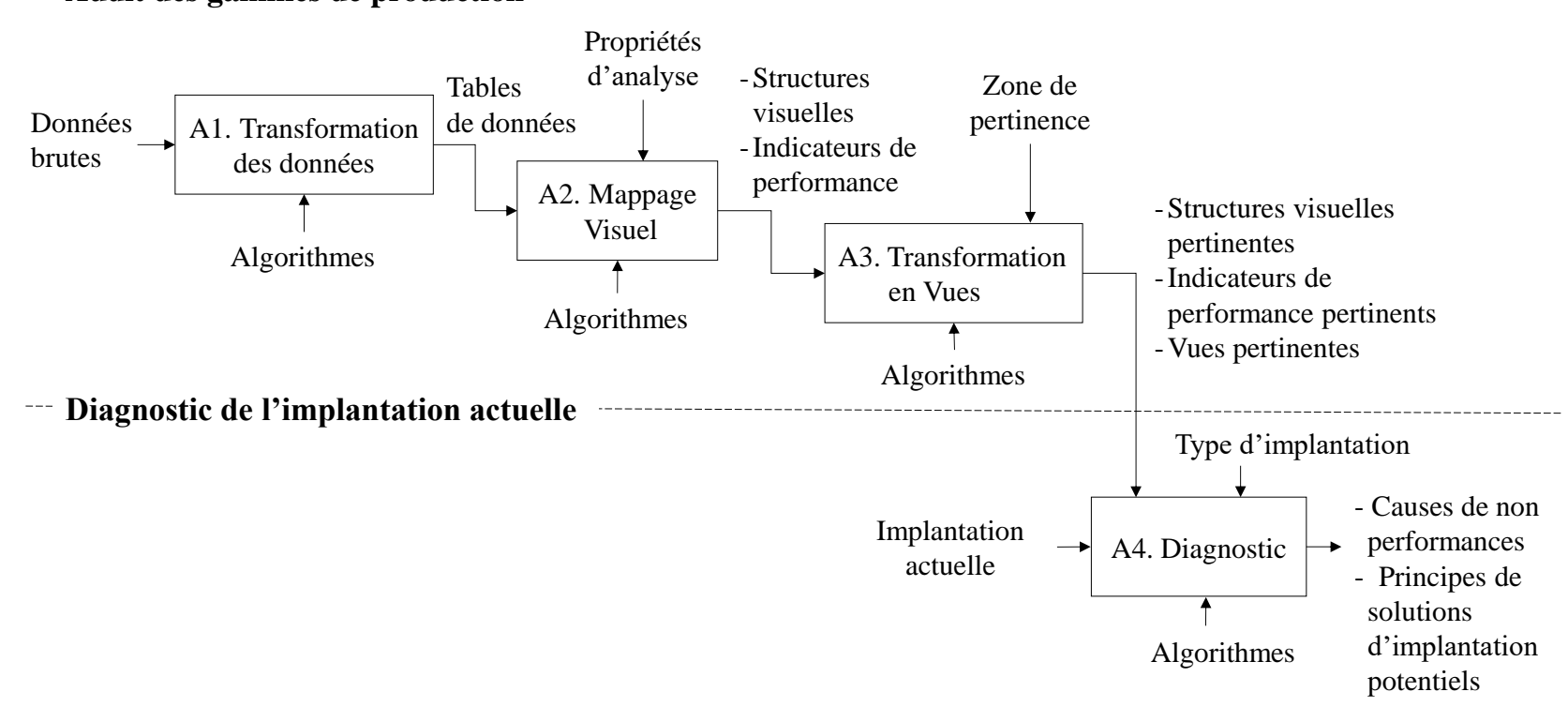

Figure 1. Phase 1 : audit des gammes et diagnostic de l'implantation du SPP 
Tableau 1. Données brutes

\begin{tabular}{|c|l|}
\hline Produits & \multicolumn{1}{|c|}{ Gammes de production } \\
\hline P01 & $\mathrm{M} 11 \rightarrow \mathrm{M} 10 \rightarrow \mathrm{M} 2 \rightarrow \mathrm{M} 1$ \\
\hline $\mathrm{P} 02$ & $\mathrm{M} 6 \rightarrow \mathrm{M} 9 \rightarrow \mathrm{M} 7 \rightarrow \mathrm{M} 5$ \\
\hline P03 & $\mathrm{M} 9 \rightarrow \mathrm{M} 4 \rightarrow \mathrm{M} 5 \rightarrow \mathrm{M} 6$ \\
\hline P04 & $\mathrm{M} 11 \rightarrow \mathrm{M} 10 \rightarrow \mathrm{M} 1 \rightarrow \mathrm{M} 2$ \\
\hline P05 & $\mathrm{M} 8 \rightarrow \mathrm{M} 3 \rightarrow \mathrm{M} 7 \rightarrow \mathrm{M} 5$ \\
\hline P06 & $\mathrm{M} 9 \rightarrow \mathrm{M} 6$ \\
\hline P07 & $\mathrm{M} 9 \rightarrow \mathrm{M} 11 \rightarrow \mathrm{M} 1$ \\
\hline P08 & M4 $\rightarrow \mathrm{M} 8 \rightarrow \mathrm{M} 3 \rightarrow \mathrm{M} 5$ \\
\hline P09 & $\mathrm{M} 11 \rightarrow \mathrm{M} 10 \rightarrow \mathrm{M} 2$ \\
\hline P10 & $\mathrm{M} 3 \rightarrow \mathrm{M} 4 \rightarrow \mathrm{M} 8 \rightarrow \mathrm{M} 5$ \\
\hline
\end{tabular}

Nombre de Produits : 10

Tableau 2. Table de données

\begin{tabular}{|c|c|c|c|c|c|c|c|c|c|c|c|}
\hline PdC/PdC & M10 & M3 & M2 & M8 & M5 & M9 & M11 & M4 & M7 & M1 & M6 \\
\hline M10 & & & 60 & & & & & & & 5 & \\
\hline M3 & & & & & 40 & & & 60 & 60 & & \\
\hline M2 & & & & & & & & & & 50 & \\
\hline M8 & & 100 & & & 60 & & & & & & \\
\hline M5 & & & & & & & & & & & 10 \\
\hline M9 & & & & & & & 10 & 10 & 10 & & 40 \\
\hline M1 & 65 & & & & & & & & & 10 & \\
\hline M4 & & & & 100 & 10 & & & & & & \\
\hline M7 & & & & & 70 & & & & & & \\
\hline M1 & & & 5 & & & & & & & & \\
\hline M6 & & & & & & 10 & & & & & \\
\hline
\end{tabular}

Nombre de Postes de Charge : 11

\section{A2. Mappage Visuel}

Les mappages sont les opérations d'analyse des tables de donnée. L'analyse consiste à décrire visuellement les tables de donnée selon un ensemble de descripteurs, appelées propriétés d'analyse par la suite. Pour chaque propriété d'analyse, des algorithmes sont appliqués. Les résultats de ces analyses sont des structures de données appelées structures visuelles et des valeurs d'indicateurs de performance.

Par exemple et à partir des données du Tableau 2 une structure visuelle d'ordre est recherchée. L'algorithme permute les lignes et les colonnes pour minimiser la quantité de flux sous la diagonale qui modélise les flux de retour en arrière. L'indicateur de performance qualifie le taux de flux de retour arrière. La structure visuelle obtenue est présentée dans le Tableau 3.
Tableau 3. Structure visuelle d'ordre

\begin{tabular}{|c|c|c|c|c|c|c|c|c|c|c|c|}
\hline Rang (PdC) & 1 & 2 & 3 & 4 & 5 & 6 & 7 & 8 & 9 & 10 & 11 \\
\hline PdC/PdC & M9 & M4 & M8 & M3 & M7 & M5 & M6 & M11 & M10 & M2 & M1 \\
\hline M9 & & 10 & & & 10 & & 40 & 10 & & & \\
\hline M4 & & & 100 & & & 10 & & & & & \\
\hline M8 & & & & 100 & & 60 & & & & & \\
\hline M3 & & 60 & & & 60 & 40 & & & & & \\
\hline M7 & & & & & & 70 & & & & & \\
\hline M5 & & & & & & & 10 & & & & \\
\hline M6 & 10 & & & & & & & & & & \\
\hline M11 & & & & & & & & & 65 & & 10 \\
\hline M10 & & & & & & & & & & 60 & 5 \\
\hline M2 & & & & & & & & & & & 50 \\
\hline M1 & & & & & & & & & & 5 & \\
\hline
\end{tabular}

\section{Indicateur de performance}

Taux de flux de retour arrière pondéré = somme des pondérations sous la diagonale / somme des pondérations totale.

$=75 / 785$

$\approx 9,6 \%$

\section{A3. Transformation en Vues}

Si une propriété d'analyse est jugée pertinente alors la structure visuelle associée est transformée en vue par des algorithmes de tracés de graphe orienté et pondéré.

Les sommets et arcs du graphe représentent respectivement les postes de charge et les flux des produits entre postes de charge. La Figure 2 représente la vue obtenue à partir de la structure visuelle d'ordre du Tableau 3. La gamme fictive est définie par la succession de l'ensemble des postes ordonnés suivant une structure d'ordre (Tableau 3, Rang $(\mathrm{PdC})$ ).

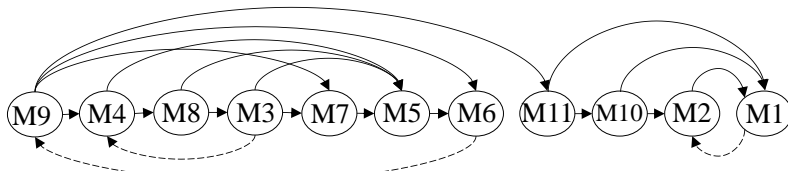

Gamme fictive : M9, M4, M8, M3, M7, M5, M6, M11, M10, M2, M1

$\longrightarrow$ Flux en avan

Flux de retour arrière

Figure 2. VUE : Relation d'ordre total entre les postes de charge

La question 1 du questionnement d'audit ci-dessus est reposée : est-ce que certains produits sont fabriqués suivant la même séquence d'opérations ? Le taux de flux de retour arrière pondéré de 9,6\% est jugé pertinent. La vue jugée pertinente en sortie 
de l'activité A3 permet au concepteur par lecture visuelle d'observer la structure d'ordre. Cette vue est une représentation qui est familière au concepteur et dans laquelle il lui est aisé d'analyser en raison du lien physique avec le plan de l'implantation actuelle (Figure 3).

\section{A4. Diagnostic de l'implantation}

Le diagnostic consiste à vérifier si l'implantation actuelle correspond à un type d'implantation.

Dans cet exemple la structure d'ordre est jugée pertinente. Le type d'implantation correspondant à la structure d'ordre est une implantation de type ligne. Le dessin de la gamme fictive sur le plan d'implantation (Figure 4) montre que les postes ne sont pas implantés en ligne. Le dessin de la gamme fictive décrit de nombreux croisements et des flux de retours en arrière, ce qui caractérise les causes de non-performance. Une implantation en ligne représente un principe de solution d'implantation potentiel pour la phase 2 d'élaboration d'un agencement conceptuel.

La vue (Figure 2) fait a priori apparaitre l'existence de 2 classes distinctes de postes de charge (" Classe 1 : M9, M4, M8, M3, M7, M5, M6 »; "Classe 2 : M11, M10, M2, M1 »). Sans le flux entre le poste de charge $M 9$ et $M 11$, les deux classes seraient indépendantes.

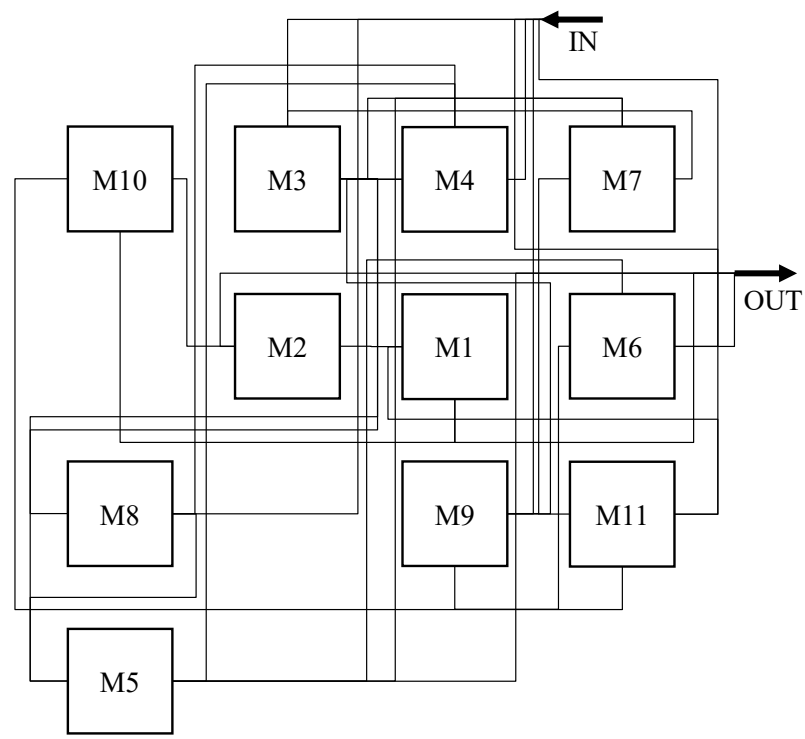

Figure 3. Plan de l'implantation actuelle et flux des produits

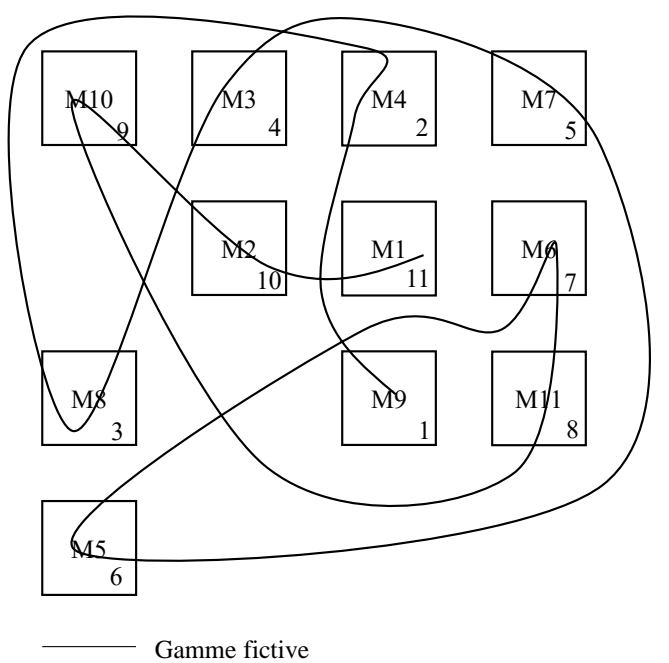

Figure 4. Représentation de la gamme fictive sur le plan de l'implantation actuelle

La question 2 du questionnement d'audit ci-dessus est reposée: est-ce que certains produits sont fabriqués sur les mêmes postes de charge ? Deux structures visuelles sont a priori combinées dans la vue, respectivement liées à la propriété d'ordre et de classe.

Le processus d'audit des gammes de production et de diagnostic de l'implantation du SPP (activités A1 à A4) peut être réitéré en considérant la propriété d'analyse de classe.

Mener la phase 1 d'audit et de diagnostic de manière intuitive comme ci-dessus peut s'avérer fastidieux et chronophage compte tenu du type de gammes considérant plusieurs milliers d'opérations. Le caractère fastidieux et chronophage ne permet pas de surveiller de manière continue d'éventuelles dérives de performance du SPP.

Afin de contribuer à systématiser et accélérer la phase 1 (Figure 1) et plus précisément l'audit des gammes de production, deux questions de recherche Q1 et Q2 sont posées :

Q1 : concernant l'activité A2 (Figure 1) quelles sont les propriétés d'analyse, les structures visuelles et indicateurs de performance à appliquer aux tables de données ?

Q2 : concernant l'activité A3 (Figure 1) quel est l'ensemble des structures visuelles et combinaison de structures visuelles potentielles à transformer en vues? 
La requête effectuée dans la littérature scientifique est relative aux mots clés extraits des questions Q1 et Q2 : gammes de production, propriétés, structures visuelles, indicateur de performance, vue. Le Tableau 4 présente les propriétés d'analyse, les structures visuelles et les indicateurs de performance recensés. Chaque propriété d'analyse est mise en correspondance avec le type d'implantation et le principe technologique du type d'implantation.

Les 7 types de relations Propriétés d'Analyse Structure Visuelle (PA-SV) recensés dans la littérature scientifique sont : 1 . ordre total, 2 . ordre partiel, 3. niveau d'antériorité, 4. relation, 5. classe, 6. source, 7. puits. Pour chaque type de propriété d'analyse, un nombre important d'algorithmes d'analyse sont proposés. Les types de propriétés d'analyse les plus étudiés concernent principalement les types "classe et ordre". La classe regroupe en famille les postes de charge ce qui permet lors de la phase d'élaboration des agencements physique du SPP de tirer profit de la similarité des séquences des opérations des différents produits pour diviser l'ensemble des postes de charge en unités de production plus petites (type d'implantation : cellule) et ainsi simplifier la gestion (De Guio et al., 1999). L'ordre permet de déterminer un " sens » dominant de flux des produits entre les postes de charge; ce qui favorise la hiérarchisation des opérations suivant les procédés (type d'implantation : ligne), et simplifie ainsi les flux (Zhou et al., 1996).

Les algorithmes utilisés pour trouver des structures visuelles à partir des propriétés d'analyse sont formalisés sous la forme de problèmes d'optimisation combinatoire. Pour chaque problème plusieurs méthodes d'optimisation sont proposées. Avec l'accroissement des vitesses de calcul durant ces 30 dernières années, ces méthodes ont trouvé leur pleine application. A titre d'illustration pour la propriété d'analyse classe, la littérature dénombre plusieurs types d'algorithmes comme : graph theorical methods (Rajagopalan, 1975), array-based methods (King, 1980), mathematical programming based methods (Kusiak, 1987; Srinivasan et al., 1990), search methods (Boctor, 1991), neural network based methods (Kaparthi \& Suresh, 1992), clustering methods (Barth \& De Guio, 1999 ; De Guio, 1999), incremental cell-formation method (Mahesh \& Srinivasan, 2002), Genetic Algorithm optimisation method (Hicks, 2005). Lee et al. (2004) ont développé 6 méthodes de recherche heuristique basées sur les algorithmes 'simulated annealing' et 'tabu search'.

Les algorithmes sont comparés par rapport au temps de calcul de recherche de solutions optimales. Yin et Yasuda (2006) développent une étude comparative des différents coefficients de similarité recensés dans les algorithmiques de construction de classe. Ils dénombrent 17 coefficients de similarité. II propose une taxonomie pour mettre en correspondance les différents coefficients de similarité.

Les travaux de la littérature étudient principalement l'application d'une propriété d'analyse à la fois. Ils décrivent la structure visuelle résultante et les indicateurs de performance associés.

Plus rarement, des travaux étudient l'application d'une combinaison de structures visuelles. Par exemple, Lee (2004) propose l'étude de l'application de la propriété d'analyse de niveau combinée avec les propriétés d'analyse de " puits et source ». Cette combinaison permet par exemple de regrouper les postes de charge de début de gammes de production (type d'implantation : zone de lancement de production) et de fin de gammes de production (type d'implantation : zone de fin de production) dans un niveau donné et ainsi simplifier la gestion des lancements et des fins de production.

Les éléments ci-dessus permettent d'apporter une réponse satisfaisante à la question Q1. Sept propriétés d'analyse, sept structures visuelles, sept indicateurs de performance et six types d'implantation ont été recensés dans les travaux de la littérature scientifique (Tableau 4). 
Tableau 4. Synthèse des résultats de la requête bibliographique

\begin{tabular}{|c|c|c|c|c|}
\hline PA-SV & Indicateur de performance & Type d'implantation & $\begin{array}{l}\text { Principe technologique du type } \\
\text { d'implantation }\end{array}$ & Références \\
\hline 1. Ordre total & $\begin{array}{l}\text { 1. Taux de flux de retour } \\
\text { arrière }\end{array}$ & \multirow[b]{2}{*}{ Type 1. Ligne } & \multirow{2}{*}{$\begin{array}{l}\text { Ordonner spatialement les } \\
\text { postes de charge selon l'ordre } \\
\text { d'utilisation des postes de } \\
\text { charge dans les gammes. }\end{array}$} & \multirow{2}{*}{$\begin{array}{l}\text { Barth et al., } 1998 \text {; De Guio et } \\
\text { al., } 1996 \text {; Irani et al., } 2000 \text {; } \\
\text { Hollier, } 1963 \text {; Huang, } 2003 \text {; } \\
\text { Sarker et al., } 2000 \text {; Zhou et al. } \\
1996\end{array}$} \\
\hline 2. Ordre partiel & 2. Taux d'ordre partiel & & & \\
\hline 3. Niveau & 3. Taux de niveau & Type 2. Niveau & $\begin{array}{l}\text { Hiérarchiser les postes de } \\
\text { charge suivant les procédés }\end{array}$ & Lee et al., 2001, 2004 \\
\hline 4. Relation & $\begin{array}{l}\text { 4. Flux pondéré entre poste } \\
\text { de charge }\end{array}$ & Type 3. Proximité & $\begin{array}{l}\text { Rapprocher les postes de } \\
\text { charge }\end{array}$ & Deb and Bhattacharyya, 2002 \\
\hline 5. Classe & $\begin{array}{l}\text { 5. Taux d'échange } \\
\text { intercellules }\end{array}$ & Type 4. Cellule & $\begin{array}{l}\text { Diviser l'ensemble des postes } \\
\text { de charge en unités de } \\
\text { production plus petites }\end{array}$ & $\begin{array}{l}\text { Barth and De Guio, } 1999 \text {; De } \\
\text { Guio et al.,1999; Mahseh et } \\
\text { al., } 2002 \text {; Kusiak., } 1987 \text {; } \\
\text { Selim et al., 1998 ; Yin et al., } \\
2006\end{array}$ \\
\hline 6. Source & 6. Taux de source & $\begin{array}{c}\text { Type } 5 . \text { Zone de lancement } \\
\text { de production }\end{array}$ & $\begin{array}{l}\text { Organiser les postes de charge } \\
\text { de début de gamme de } \\
\text { production }\end{array}$ & \multirow{2}{*}{$\begin{array}{l}\text { Bard et al., } 1989 \text {; De Guio et } \\
\text { al., } 1996 \text {; Lee et al., 2001, } \\
2004 \text {; Zhou et al., } 2003\end{array}$} \\
\hline 7. Puits & 7. Taux de puits & $\begin{array}{c}\text { Type 6. Zone de fin de } \\
\text { production }\end{array}$ & $\begin{array}{l}\text { Organiser les Postes de Charge } \\
\text { de fin de gamme de production }\end{array}$ & \\
\hline
\end{tabular}

Les éléments ci-dessus ne permettent pas d'apporter une réponse complètement satisfaisante à la question Q2. Les combinaisons entre structures visuelles sont très peu étudiées et explicitées. Pour mettre en évidence la ou les propriétés dominantes ou distinctives des tables de données, la juxtaposition des vues mono-structure visuelle ne suffit pas pour décrire des vues multi- structures visuelles dues au fait de leurs combinaisons.

La prise en charge dans la vue de combinaisons de structures visuelles a pour objet de faire émerger des caractéristiques nouvelles (Card, 1999). Tenir compte simultanément des 7 relations PA-SV (Tableau 4) recensées revient à étudier un modèle générique de 127 structures visuelles et combinaisons de structures visuelles (1).

$C_{7}^{1}+C_{7}^{2}+C_{7}^{3}+C_{7}^{4}+C_{7}^{5}+C_{7}^{6}+C_{7}^{7}=127$

II est difficilement concevable de mener 127 analyses des tables de données à partir de 127 combinaisons de structures visuelles transformées en 127 vues.

En résumé et à notre connaissance il n'existe pas de modèle de référence pour l'audit des gammes de production
L'objectif de ce papier est de tester les potentialités d'une démarche d'analyse combinatoire des structures visuelles selon les principes organisationnels du lean manufacturing.

La contribution attendue est la création d'un modèle de référence des structures visuelles et de leurs combinaisons potentiellement pertinentes à transformer en vue. Ce modèle de référence doit permettre l'automatisation de l'audit des gammes de production.

\section{CONCEPTION DU MODÈLE DE RÉFÉRENCE}

Le modèle de référence est conçu en trois étapes (Figure 5).

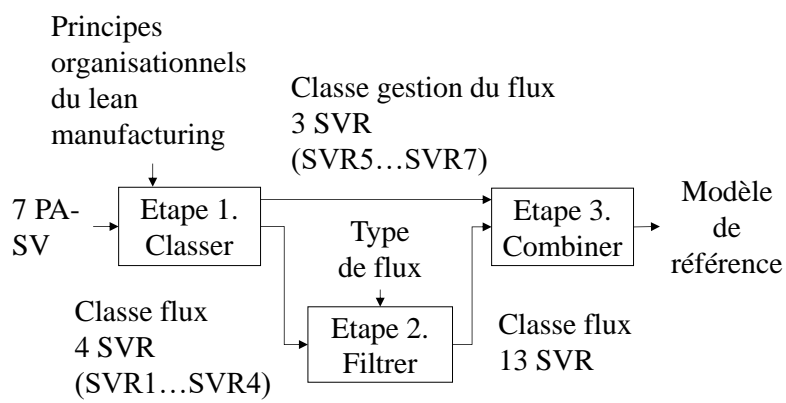

Figure 5. Conception du modèle du référence (IDEFO) 


\subsection{ETAPE 1. Classer}

La formalisation des principes technologiques des types d'implantation associés aux propriétés (Tableau 4) permet d'identifier les deux principes organisationnels du lean manufacturing lié au flux des produits : principe 1. simplifier le flux des produits ; principe 2. simplifier la gestion du flux des produits. L'étape 1 consiste à classer les structures visuelles (Tableau 4) selon ces 2 principes indissociables, mais conceptuellement distincts. Les 2 classes sont respectivement nommées : classe flux (principe 1) et classe gestion du flux (principe 2). Les classes flux et gestion du flux sont respectivement présentées dans le Tableau 5 et le Tableau 6. Les classes flux et gestion du flux comportent respectivement 4 et 3 structures visuelles, appelées par la suite des structures visuelles de référence (SVR).

Tableau 5. Classe flux

\begin{tabular}{|c|c|c|c|c|}
\hline Type \# & \multicolumn{2}{|c|}{$\begin{array}{c}\text { Type 1 } \\
\text { Ligne }\end{array}$} & $\begin{array}{c}\text { Type 2 } \\
\text { Niveau }\end{array}$ & $\begin{array}{c}\text { Type 3 } \\
\text { Proximité }\end{array}$ \\
\hline \# PA-S V & $\begin{array}{c}\text { 1. Ordre } \\
\text { Total }\end{array}$ & $\begin{array}{c}\text { 2. Ordre } \\
\text { Partiel }\end{array}$ & 3. Niveau & $\begin{array}{c}4 . \\
\text { Relation }\end{array}$ \\
\hline SVR \# & SVR 1 & SVR 2 & SVR 3 & SVR 4 \\
\hline $\begin{array}{c}\text { Intitulé } \\
\text { SVR }\end{array}$ & $\begin{array}{c}\text { Ordre } \\
\text { total }\end{array}$ & $\begin{array}{c}\text { Ordre } \\
\text { partiel }\end{array}$ & $\begin{array}{c}\text { Graphe } \\
\text { de niveau }\end{array}$ & Relation \\
\hline
\end{tabular}

La classe flux doit prendre en compte la globalité du flux des produits entre postes de charge (Burbidge, 1989 ; Tompkins et al., 2003 ; Zhou et al., 2003). Ceci est essentiel puisque la phase d'élaboration des agencements physique du SPP exige le positionnement de l'ensemble des postes de charge sur la surface géométrique dédiée au SPP.

La classe gestion du flux doit permettre d'identifier et de décrire des éléments de gestion du flux des produits relatifs à des postes de charge. Ceci est essentiel puisque ces postes de charge requièrent un positionnement et un type de gestion particulier dans l'implantation. Concernant les propriétés source et le puits, deux types de gestion sont distingués (Tableau 6) : une gestion centralisée et une gestion distribuée respectivement pour les zones de lancement (type d'implantation 5.1 et 5.2) et de fin de production (type d'implantation 6.1 et $6.2)$.

Tableau 6. Classe gestion du flux

\begin{tabular}{|c|c|c|c|c|c|}
\hline \multirow[b]{2}{*}{ Type \# } & \multirow[b]{2}{*}{$\begin{array}{l}\text { Type } 4 \\
\text { Cellule }\end{array}$} & \multicolumn{2}{|c|}{$\begin{array}{c}\text { Type } 5 . \text { Zone de lancement } \\
\text { de production }\end{array}$} & \multicolumn{2}{|c|}{$\begin{array}{l}\text { Type } 6 . \text { Zone de fin de } \\
\text { production }\end{array}$} \\
\hline & & $\begin{array}{l}\text { Type 5.1 } \\
\text { Zone de } \\
\text { lancement } \\
\text { centralisée }\end{array}$ & \begin{tabular}{|l} 
Type 5.2 \\
Zone de \\
lancement \\
distribuée
\end{tabular} & \begin{tabular}{|l|} 
Type 6.1 \\
Zone de fin de \\
production \\
centralisée
\end{tabular} & $\begin{array}{l}\text { Type } 6.1 \\
\text { Zone de fin } \\
\text { de production } \\
\text { distribuée }\end{array}$ \\
\hline \# PA-SV & 5. Classe & \multicolumn{2}{|c|}{ 6. Source } & \multicolumn{2}{|c|}{ 7. Puits } \\
\hline SVR \# & SVR 5 & \multicolumn{2}{|c|}{ SVR 6} & \multicolumn{2}{|c|}{ SVR 7} \\
\hline $\begin{array}{c}\text { Intitulé } \\
\text { SVR }\end{array}$ & Classe & \multicolumn{2}{|c|}{ Source } & \multicolumn{2}{|c|}{ Puits } \\
\hline
\end{tabular}

\subsection{ETAPE 2. Filtrer}

L'étape 2 consiste à filtrer les SVR de la classe flux selon les types de flux des produits : flux de retour arrière, flux transitifs, flux direct (Sarker et $\mathrm{Xu}, 2000$ ). Ce filtrage permet de mettre en évidence des connaissances par type de flux ou par des combinaisons de types de flux. Les deux types de flux considérés pour le filtrage sont : les flux transitifs et les flux de retour arrière. Sarker et Xu (2000) qualifient ces deux types de flux : " compliquent le flux ", "perturbent le sens du flux ». "I n'est pas étonnant de retrouver ces deux facteurs de bruit dans l'expression des indicateurs de performance associés aux structures visuelles. Les 4 SVR de la classe flux sont filtrées à l'aide de ces 2 types de déplacements. Après filtrage la classe flux contient 13 SVR (Tableau 7) : 4 SVR (SVR1, SVR2, SVR3, SVR4) sans filtrage et 9 SVR (SVR ** avec filtrage).

\subsection{ETAPE 3. Combiner}

L'étape 3 consiste à combiner les 13 SVR de la classe flux avec les 3 SVR de la classe gestion du flux. II en résulte un modèle de référence de 13 vues de référence (VUER) (Tableau 7). La partie grisée indique les combinaisons de SVR à transformer en vue.

A titre d'illustration, la VUER2 combine les structures visuelles suivantes : (classe flux: ordre total acyclique) $X$ (classe gestion du flux : source et puits). 
Tableau 7. Modèle de référence

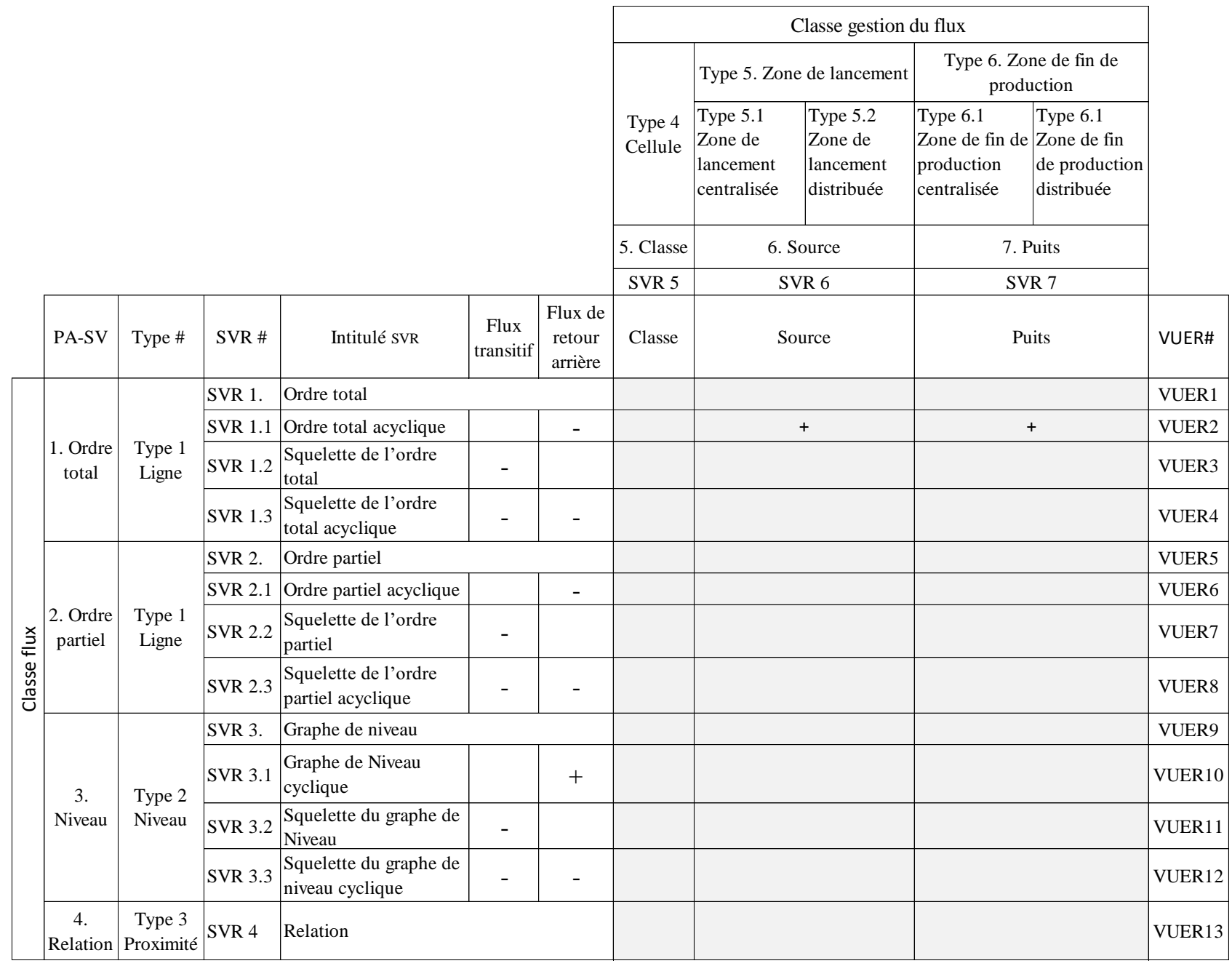

't' visualiser ; '-' ne pas visualiser

\section{INSTANCIATION DU MODELE DE REFERENCE}

Cette section décrit la règle d'instanciation du modèle de référence pour extraire un modèle particulier. Pour instancier le modèle de référence, la règle suivante est appliquée: si la propriété d'analyse est jugée pertinente, alors les structures visuelles associées sont à transformer en vue (Figure 1 , activité $A 3)$.

\section{Notation :}

$\mathrm{j}:$ un indicateur de Performance

$\mathrm{Vj}$ : valeur de $\mathrm{j}$

VOj : valeur optimale de $j$

lj : domaine de définition de $\mathrm{j}$

$\operatorname{lnf}(\mathrm{lj})$ : valeur inférieure de lj

$\operatorname{Sup}(\mathrm{l} j)$ : valeur supérieure de $\mathrm{l} j$

$\mathrm{Pj}$ : fonction de pertinence relative à $\mathrm{j}$ $\mathrm{q}_{\mathrm{j}}$ : seuil de pertinence de $\mathrm{j}$

$\mathrm{Zj}:$ zone de pertinence de $\mathrm{j}$

$\operatorname{Inf}(Z j)$ : valeur inférieure de $Z$ de $j$

$\operatorname{Sup}(Z j)$ : valeur supérieure de $Z$ de $j$

$\mathrm{dj}$ : distance entre VOj et $\mathrm{Vj}$

Pour évaluer la pertinence des propriétés d'analyse, une zone de pertinence $\mathrm{Zj}:[\operatorname{Inf}(\mathrm{Zj}), \operatorname{Sup}(\mathrm{Zj})]$ est définie pour chaque indicateur de performance $j$ (Tableau 4) relatif à chaque propriété d'analyse. Cette zone de pertinence $Z \mathrm{Jj}$ est définie comme suit :

$\mathrm{Zj} \subset \mathrm{Ij}$

VOj $\in[\operatorname{Inf}(\mathrm{Ij}), \operatorname{Sup}(\mathrm{Ij})]$

$\operatorname{Inf}(\mathrm{Zj})=\left\{\begin{array}{l}\operatorname{VOj}-\text { qj ssi VOj }- \text { qj } \geq \operatorname{Inf}(\mathrm{Ij}) \\ \operatorname{Inf}(\mathrm{Ij}) \text { ssi VOj }- \text { qj }<\operatorname{Inf}(\mathrm{Ij})\end{array}\right.$

$\operatorname{Sup}(Z j)=\left\{\begin{array}{l}\operatorname{VOj}+q j \operatorname{ssi} V O j+q j \leq \operatorname{Sup}(\mathrm{Ij}) \\ \operatorname{Sup}(\mathrm{Ij}) \text { ssi VOj }+ \text { qj }>\operatorname{Inf}(\mathrm{Ij})\end{array}\right.$ 
La définition du seuil de pertinence $\mathrm{q}_{\mathrm{j}}$ dépend de plusieurs facteurs comme l'expérience du concepteur en charge de l'étude d'implantation, le contexte de l'étude menée, le secteur d'activité, les produits, les postes de charge.

Si la valeur de l'indicateur de performance $\mathrm{Vj}$ associée à la propriété $A$ est incluse dans la zone de pertinence $\mathrm{Zj}$ de $\mathrm{A}$ alors $\mathrm{A}$ est jugée pertinente.

Pour savoir comment se situe la valeur de Vj par rapport à VOj, on peut :

Définir dj comme suit : $d j=|V O j-V j|$

Et considérer la fonction Pj comme suit :

$\operatorname{Pj}(\mathrm{dj})=\left\{\begin{array}{llll}1 & \text { ssi } & \text { dj } \leq \mathrm{q} & \text { Pertinence }(+) \\ 0 & \text { ssi } & \text { dj }>\mathrm{q} & \text { Non Pertinence }\end{array}\right.$

$\operatorname{Pj}(\mathrm{dj})$ est une fonction de $[\operatorname{Inf}(I), \operatorname{Sup}(\mathrm{I})] \operatorname{sur}\{0,1\}$.

Le Tableau 8 précise pour chaque indicateur: la formule, le domaine de définition (I) et la valeur optimale (VOj). Dans le cas de la PA-SV source et puits, deux VOj sont précisés pour tenir compte des deux types de gestion des flux centralisés et décentralisés.
Tableau 8. Indicateurs de performance, formule, domaine de définition, valeur optimale

\begin{tabular}{|c|c|c|c|c|c|c|}
\hline PA-SV & $\begin{array}{c}\text { Indicateur de } \\
\text { performance } \\
\text { IP(j) }\end{array}$ & Formule & \begin{tabular}{|c} 
Domaine \\
de \\
définition \\
{$[\%](I)$}
\end{tabular} & $\begin{array}{c}\text { Type } \\
\text { d'implantation }^{2}\end{array}$ & $\begin{array}{c}\text { Valeur } \\
\text { Optimale } \\
\text { [\%] (VOj) }\end{array}$ & Référence \\
\hline $\begin{array}{l}1 . \\
\text { Ordre } \\
\text { total }\end{array}$ & \begin{tabular}{|l|} 
IP(1) \\
Taux de flux \\
de retour \\
arrière [\%]
\end{tabular} & $\begin{array}{l}\Sigma \text { (flux de } \\
\text { retours } \\
\text { arrière) / F }\end{array}$ & {$[0,100[$} & & 0 & \\
\hline $\begin{array}{l}2 . \\
\text { Ordre } \\
\text { partiel }\end{array}$ & $\begin{array}{l}\text { IP(2) } \\
\text { d'ordre partiel } \\
{[\%]}\end{array}$ & $\begin{array}{l}\text { Nombre de } \\
\text { postes de } \\
\text { charge ayant } \\
\text { plus qu'un } \\
\text { rang possible) } \\
\text { / C }\end{array}$ & {$[0,100]$} & Ligne & 100 & $\begin{array}{l}\text { Zhou et } \\
\text { al., } 1996\end{array}$ \\
\hline $\begin{array}{l}3 . \\
\text { Niveau }\end{array}$ & \begin{tabular}{|l}
$\mathrm{IP}(3)$ \\
Taux de \\
niveau [\%]
\end{tabular} & $\begin{array}{l}\text { Nombre de } \\
\text { niveaux / C }\end{array}$ & $\begin{array}{l}{[100 / \mathrm{C}, 1} \\
00]\end{array}$ & Niveau & $100 / \mathrm{C}$ & $\begin{array}{l}\text { Kahn, } \\
1962\end{array}$ \\
\hline $\begin{array}{l}4 . \\
\text { Relation }\end{array}$ & $\begin{array}{l}\text { IP( } 4) \\
\text { Flux pondéré } \\
\text { entre postes } \\
\text { de charge. }\end{array}$ & \begin{tabular}{|l} 
Test \\
d'adéquation \\
loi de Pareto ; \\
Test de \\
Kolmogorov- \\
Smirnov
\end{tabular} & $\{0 ; 1\}$ & Proximité & 1 & \begin{tabular}{|l} 
Frank \& \\
Massey, \\
1951
\end{tabular} \\
\hline $\begin{array}{l}5 . \\
\text { Classe }\end{array}$ & \begin{tabular}{|l} 
IP(5) \\
Taux \\
d'échange \\
intercellules \\
{$[\%]$}
\end{tabular} & $\begin{array}{l}\Sigma \text { (flux inter } \\
\text { cellules) / F }\end{array}$ & {$[0,50[$} & Cellule & 0 & $\begin{array}{l}\text { De Guio, } \\
1999\end{array}$ \\
\hline \multirow{2}{*}{$\begin{array}{l}6 . \\
\text { Source }\end{array}$} & \multirow{2}{*}{$\begin{array}{l}\operatorname{IP}(6) \\
\text { Taux de } \\
\text { source [\%] }\end{array}$} & \multirow{2}{*}{$\begin{array}{l}\text { Nombre de } \\
\text { sources / C }\end{array}$} & \multirow{2}{*}[0,100]{} & \begin{tabular}{|l} 
Zone de \\
lancement de \\
production \\
centralisée \\
\end{tabular} & $100 / \mathrm{C}$ & \multirow{4}{*}{ I } \\
\hline & & & & \begin{tabular}{|l|} 
Zone de \\
lancement de \\
production \\
distribuée \\
\end{tabular} & 100 & \\
\hline \multirow{2}{*}{$\begin{array}{l}7 . \\
\text { Puits }\end{array}$} & \multirow{2}{*}{$\begin{array}{l}\operatorname{IP}(7) \\
\text { Taux de puits } \\
{[\%]}\end{array}$} & \multirow{2}{*}{$\begin{array}{l}\text { Nombre de } \\
\text { puits / C }\end{array}$} & \multirow{2}{*}[0,100]{} & \begin{tabular}{|l|} 
Zone de fin de \\
production \\
centralisée
\end{tabular} & $100 / \mathrm{C}$ & \\
\hline & & & & \begin{tabular}{|l|} 
Zone de fin de \\
production \\
distribuée
\end{tabular} & 100 & \\
\hline
\end{tabular}

$\mathrm{C}$ : nombre de postes de charge ; $\mathrm{F}$ : somme des flux entre postes de charge.

\section{VALIDATION INDUSTRIELLE DU MODELE DE REFERENCE}

Les deux questions de validation du modèle de référence sont les suivantes:

Q3 : Peut-on confirmer par l'expérimentation la pertinence des propriétés d'analyse recensées dans la littérature scientifique?

Q4 : Peut-on confirmer par l'expérimentation la représentativité du modèle de référence?

Pour répondre à ces questions, l'expérimentation suivante est menée. Le processus d'audit des gammes de production (A1. Transformation des données, A2. Mappage visuel, A3. Transformation en vues) est appliqué sur 19 cas industriels. Afin d'opérationnaliser la validation du modèle de référence, le processus d'audit des gammes est automatisé. L'objectif est d'extraire automatiquement un modèle particulier relatif à 
chaque cas industriel. À partir des données brutes d'un cas industriel particulier, les résultats suivants sont générés : les structures visuelles, les indicateurs de performance et l'affichage des vues pertinentes.

Les 19 cas industriels considérés sont issus des secteurs d'activités de l'aéronautique, l'automobile et l'équipement électrique. Le Tableau 9 présente une description des 19 cas industriels (C1 à C19) à partir des gammes industrielles de production (données brutes). Pour chaque cas, le nombre de postes de charge, le nombre des produits et le nombre d'opérations sont décrits.
Par souci de concision, seuls les résultats suivants sont présentés : calcul des indicateurs de performances considérés (Tableau 10), calcul de la pertinence des propriétés d'analyse (Tableau 11). Le Tableau 10 présente les valeurs des indicateurs de performance associés aux propriétés d'analyse. Les formules de calcul employées sont issues du Tableau 8.

Le Tableau 11 présente pour chaque relation PA-SV, le type d'implantation considéré, le seuil de pertinence, la zone de pertinence et le résultat de calcul de pertinence.

Tableau 9. Description des 19 cas industriels (données brutes)

\begin{tabular}{|c|c|c|c|c|c|c|c|c|c|c|c|c|c|c|c|c|c|c|c|}
\hline & \multicolumn{19}{|c|}{ Cas indutriels $(\mathrm{C} 1 \ldots \mathrm{C} 19)$} \\
\hline & $\mathrm{C} 1$ & $\mathrm{C} 2$ & C3 & $\mathrm{C} 4$ & $\mathrm{C} 5$ & C6 & $\mathrm{C} 7$ & $\mathrm{C} 8$ & C9 & $\mathrm{C} 10$ & C11 & C12 & $\mathrm{C} 13$ & C14 & $\mathrm{C} 15$ & $\mathrm{C} 16$ & $\mathrm{C} 17$ & $\mathrm{C} 18$ & C19 \\
\hline $\begin{array}{l}\text { Nombre de } \\
\text { postes de charge }\end{array}$ & 17 & 40 & 16 & 28 & 26 & 46 & 19 & 73 & 87 & 14 & 12 & 104 & 29 & 21 & 90 & 43 & 31 & 23 & 25 \\
\hline $\begin{array}{l}\text { Nombre de } \\
\text { produits }\end{array}$ & 30 & 30 & 18 & 2 & 32 & 19 & 206 & 47 & 47 & 51 & 7 & 183 & 3019 & 2262 & 110 & 110 & 85 & 85 & 85 \\
\hline $\begin{array}{l}\text { Nombre } \\
\text { d'opérations }\end{array}$ & 310 & 864 & 528 & 527 & 1075 & 946 & 1386 & 9240 & 8910 & 864 & 132 & 4246 & 7992 & 3690 & 11017 & 4368 & 2256 & 996 & 1880 \\
\hline
\end{tabular}

Tableau 10. Résultats de calcul des indicateurs de performance (Activité A2)

\begin{tabular}{|c|c|c|c|c|c|c|c|c|c|c|c|c|c|c|c|c|c|c|c|c|}
\hline \multirow[b]{2}{*}{ PA-SV } & \multirow{2}{*}{$\begin{array}{l}\text { Indicateur de } \\
\text { Performance } \\
\text { IP(j) }\end{array}$} & \multicolumn{19}{|c|}{ Cas indutriels (C1 ...C19) } \\
\hline & & $\mathrm{C} 1$ & $\mathrm{C} 2$ & C3 & $\mathrm{C} 4$ & C5 & C6 & C7 & C8 & C9 & C10 & C11 & C12 & C13 & C14 & C15 & C16 & C17 & C18 & C19 \\
\hline 1. Ordre total & IP1 & 1 & 3 & 0 & 9 & 2 & 14 & 2 & 31 & 31 & 11 & 0 & 13 & 5 & 5 & 18 & 24 & 22 & 20 & 20 \\
\hline 2. Ordre partiel & IP2 & 76 & 69 & 82 & 64 & 77 & 71 & 42 & 79 & 81 & 35 & 16 & 81 & 58 & 52 & 71 & 62 & 74 & 39 & 88 \\
\hline 3. Niveau & IP3 & 47 & 52 & 19 & 50 & 42 & 46 & 74 & 81 & 18 & 80 & 83 & 15 & 68 & 72 & 46 & 63 & 13 & 48 & 48 \\
\hline 4. Relation & IP4 & 0 & 0 & 0 & 1 & 0 & 1 & 0 & 1 & 1 & 0 & 0 & 0 & 0 & 1 & 1 & 1 & 1 & 1 & 0 \\
\hline 5. Classe & IP5 & 16 & 8 & 20 & 11 & 14 & 10 & 10 & 40 & 12 & 12 & 3 & 20 & 40 & 9 & 11 & 19 & 22 & 32 & 31 \\
\hline 6. Source & IP6 & 18 & 21 & 10 & 9 & 27 & 4 & 11 & 4 & 2 & 14 & 8 & 15 & 22 & 29 & 3 & 19 & 29 & 26 & 18 \\
\hline 7. Puits & IP7 & 18 & 5 & 8 & 15 & 22 & 9 & 52 & 16 & 14 & 7 & 8 & 44 & 38 & 43 & 20 & 7 & 19 & 22 & 28 \\
\hline
\end{tabular}


Tableau 11. Résultats de calcul de pertinence des propriétés d'analyse (Activité A3)

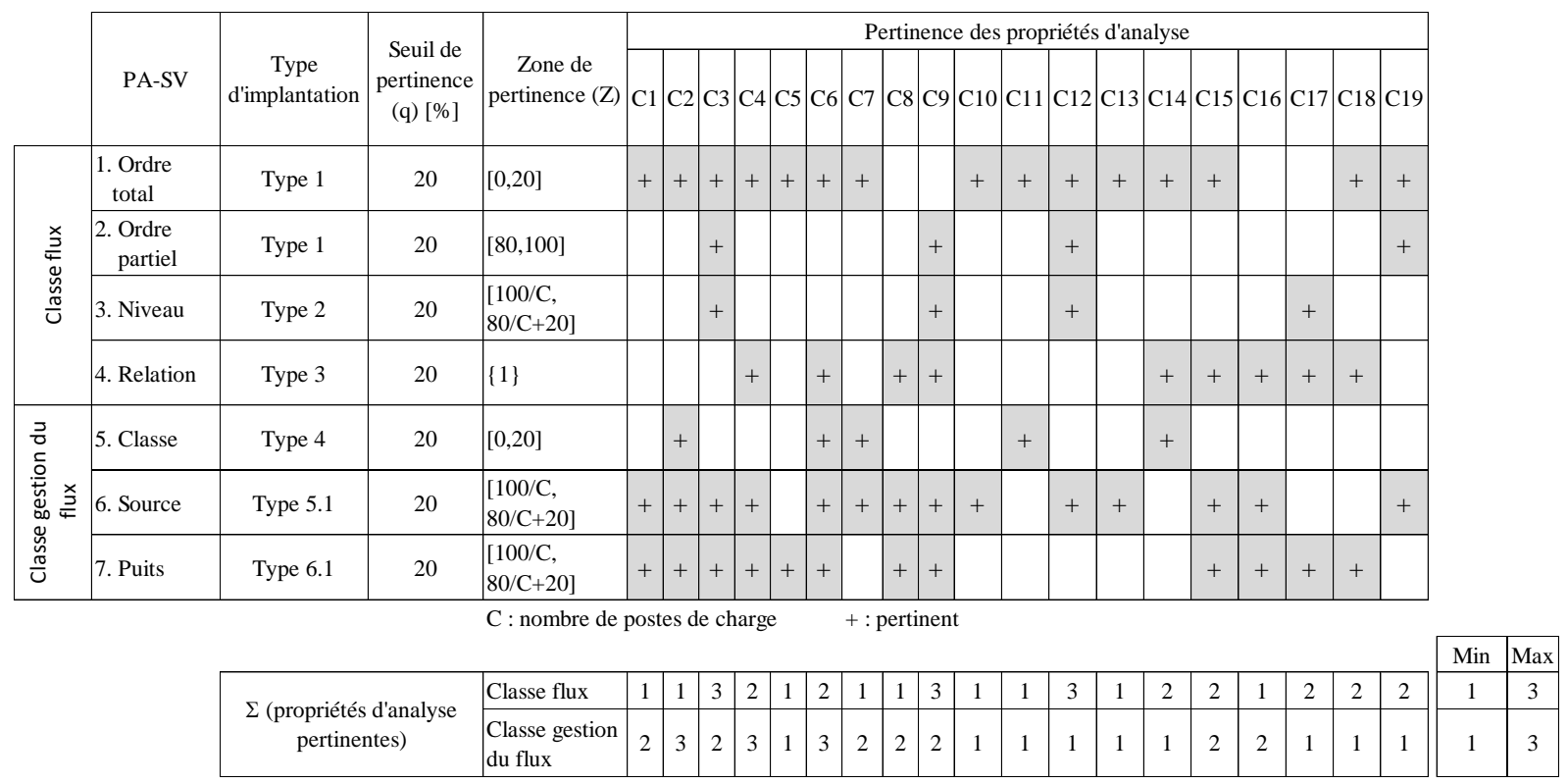

Les résultats du Tableau 11 mettent en exergue les observations suivantes:

Par ligne :

Aucune ligne n'est vide : chaque propriété d'analyse est jugée pertinente au moins une fois.

Aucune ligne n'est complète : la pertinence des propriétés d'analyse est contingente (Damand et al., 2021).

Par colonne :

Aucune colonne n'est vide : chaque cas industriel est décrit par au moins une propriété de la classe flux et deux propriétés d'analyse de la classe gestion de flux: classe flux $(\min =1)$; classe gestion du flux (min=1).

Aucune colonne n'est complète : dans aucun cas, toutes les propriétés d'analyses sont jugées pertinentes : classe flux $(\max =3)$; classe gestion du flux $(\max =3)$.

Les observations ci-dessus sont liées à la fois à la particularité des cas industriels étudiés et aux seuils de pertinence considérés par propriétés d'analyse. Le seuil de pertinence qj est fixé pour l'expérimentation à $20 \%$ de la longueur du domaine de définition lj pour toutes les propriétés d'analyse. Ce seuil strict est aligné avec l'objectif de mettre en évidence les propriétés dominantes et distinctives des tables de données.
Exemple du calcul du seuil de pertinence (q) et de la zone de pertinence (Z) : pour I'IP1, (I: [0, 100[, V0j=0, $q=20(20=20 \%|I:[0,100]|, Z:[0,20])$.

Les résultats représentent une capitalisation de connaissances et non une signification statistique d'une population de cas industriels. Le nombre de cas industriels est relatif. En effet, deux cas suffisent pour montrer l'existence potentielle de la contingence des propriétés d'analyse.

En réponse respectivement aux questions Q3 et Q4 : La pertinence des 7 propriétés d'analyse est confirmée. Cependant, la pertinence des propriétés d'analyse est contingente.

La représentativité du modèle de référence est confirmée. Chaque cas industriel a pu être caractérisé par des structures visuelles de référence.

\section{CONCLUSION}

L'audit et le diagnostic de l'implantation du SPP sont considérés dans ce papier. Le point de vue considéré est le point de vue du flux physique des produits modélisé dans les gammes de production. Le processus d'audit des gammes de production est usuellement décomposé en trois activités : activité A1. transformation de données, activité A2. mappage visuel, activité $A 3$. transformation en vue. 
Afin de mettre en œuvre ce processus d'audit, 2 questions de recherche sont posées : (Q1) concernant l'activité 2 , quels sont les propriétés d'analyse, les structures visuelles et indicateurs de performance à appliquer aux tables de données ? ; (Q2) concernant l'activité 3, Figure 1, quel est l'ensemble des structures visuelles et combinaisons de structures visuelles potentielles à transformer en vues?

La revue de la littérature apporte une réponse acceptable à Q1. Sept propriétés d'analyse, sept structures visuelles, sept indicateurs de performance et six types d'implantation sont recensés. En revanche, la revue de la littérature apporte une réponse incomplète à Q2. La contribution de ce papier consiste alors à tester les potentialités d'un modèle de référence des structures visuelles. La structuration du modèle de référence est basée sur une taxonomie des structures visuelles selon 2 principes organisationnels du lean manufacturing (simplification du flux des produits $X$ simplification de la gestion de flux du flux des produits). Le modèle de référence combine 13 structures visuelles de référence liées au flux des produits avec 3 structures visuelles de référence liées à la gestion du flux des produits. Le modèle de référence compte 13 vues de référence.

La validation du modèle de référence soulève deux questions : (Q3) Peut-on confirmer par l'expérimentation la pertinence des propriétés d'analyse recensées dans la littérature scientifique? (Q4) Peut-on confirmer par l'expérimentation la représentativité du modèle de référence ?

L'expérimentation menée consiste à décliner le processus d'audit des gammes dans le cadre de données brutes issues de 19 cas industriels. L'expérimentation permet a priori de valider le modèle de référence. D'une part, en réponse à Q3, les observations révèlent que la pertinence des propriétés d'analyses est contingente. D'autre part, en réponse à $\mathrm{Q} 4$, les observations révèlent que chacun des cas industriels est caractérisé par des SVR. A noter que dans le cadre de l'expérimentation le nombre de VUER varie entre 1 VUER et 8 VUER.
Le nombre de VUER est corrélé avec la valeur du seuil de pertinence des propriétés d'analyse. Moins le seuil de pertinence est strict, et, plus le nombre de VUER est important avec un maximum de 13 VUER. La compréhension du modèle de référence par le concepteur reste cognitivement acceptable.

La perspective de ce travail concerne l'activité de diagnostic du SPP (activité 4, Figure 1). L'implantation est-elle implantée selon tel type d'implantation? Quels sont les causes de nonperformance ? Quels sont les principes de solutions à considérer?

Afin d'intégrer le diagnostic 4.0 dans un système proactif de surveillance des dérives de la performance du SPP, la contribution attendue consiste à formaliser des indicateurs de mesure du degré de concordance entre les propriétés contenues dans les gammes de production et les propriétés contenues dans le plan d'implantation.

\section{REMERCIEMENTS}

Cette recherche est réalisée dans le cadre du projet Offensive Sciences numéro 3.11 « Virtual Innovative Real Time Factory " (VIRTFac) qui bénéficie du soutien financier de l'Offensive Sciences de la Région Métropolitaine Trinationale du Rhin Supérieur, du programme INTERREG $V$ Rhin Supérieur et du Fonds européen de développement régional (FEDER) de l'union européenne.

\section{REFERENCES}

Alfnes, E., \& Martinsen, K. (2006, July 25-28). Modeling and design of flow manufacturing systems for SMEs [Paper presentation]. Proceedings of the 5th 3rd CIRP International Seminar on Intelligent Computations in Manufacturing Engineering, Ischia, Italy.

Amoako-Gyampah, K., \& Acquaah, M. (2008). Manufacturing Strategy, Competitive Strategy, and Firm Performance: An Empirical Study in a Developing Economic Environment. International Journal of Production Economics, 111(2), 575-92. https://doi.org/10.1016/j.ijpe.2007.02.030

Bard, J.F., \& Feo T.A. (1989). Operations Sequencing in Discrete Parts Manufacturing. Management Science, 35(2), 249-255. https://doi.org/10.1287/mnsc.35.2.249 
Barth, M., \& De Guio R. (1999). Industrial Implementation of Production Flow Analysis. In IRANI S.A. (ed.) Handbook of Cellular Manufacturing Systems, John Wiley \& Sons., (pp. 497-527). https://doi.org/10.1002/9780470172476.ch16

Barth, M., De Guio R., \& Zhou J. (1998, August 9-12). A graph theoretic approach for the production line formation problem. Proceeding of the $2^{\text {nd }}$ International Conference on Engineering Design and Automation, Maui, Hawaii.

Boctor, F.F. (1991). A linear formulation of the machine-part cell formation problem. International Journal of Production Research, 29(2), 343-356. https://doi.org/10.1080/00207549108930075

Burbidge, J.L. (1989). Production flow analysis for planning group technology. Journal of Operations Management, 10(1), 5-27. https://doi.org/10.1016/0272-6963(91)90033-T

Card, S.K., Mackinlay, J.D., \& Shneiderman, B. (1999). Readings in Information Visualization: Using Vision to Think. San Francisco, California: Morgan-Kaufmann Publishers.

CHANEGRIH, T., \& CREUSIER, J. (2015). Le lean manufacturing dans l'industrie française : états des lieux et implications pratiques. Revue Française De Gestion Industrielle, 34(4), 59-71. https://doi.org/10.53102/2015.34.04.831

Chi, E.H. (2000, October 9-10). Taxonomy of Visualization Techniques Using the Data State Reference Model. Proceedings of the IEEE Symposium on Information Visualization 2000. INFOVIS 2000, Salt Lake City, UT, USA. https://doi.org/10.1109/INFVIS.2000.885092

Damand, D., Gamoura, S., Barth, M., 'Maturité du système physique de production : modèle de diagnostic 4.0', CIGI-Qualita21 : Conférence Internationale Génie Industriel QUALITA- Grenoble, 5-7 mai 2021.

De Guio R., Barth, M. \& Zhou, J. (1996, July 9-12). Checking Algorithms for the Weighted Feedback Arc Set problem with Order Constraints. Proceedings of the Symposium on modelling, analysis and simulation: CESA'96 IMACS multiconference, Computational engineering in systems applications, Lille, France.

De Guio, R., \& Barth, M. (1999). Cell Formation Using Production Flow Analysis. In IRANI S.A. (ed.) Handbook of Cellular Manufacturing Systems, John Wiley \& Sons., (pp. 69-111). https://doi.org/10.1002/9780470172476.ch16

Deb, S.K. \& Bhattacharyya, B. (2002). Fuzzy decision support system for manufacturing facilities layout planning. Decision Support Systems, 40(2), 305- 314. https://doi.org/10.1016/i.dss.2003.12.007
Frank, J. \& Massey, J.R. (1951). The KolmogorovSmirnov Test for Goodness of Fit. Journal of the American Statistical Association, 46(253), 68-78. https://doi.org/10.1080/01621459.1951.10500769

Hicks, C. (2004). A Genetic Algorithm tool for designing manufacturing facilities in the capital goods industry. International Journal of Production Economics, 90(2), 199-211. https://doi.org/10.1016/S09255273(02)00467-X

Hollier, R.H. (1963). The layout of Multi-Product Lines. International Journal of Production Research, 2(1), 47-57. https://doi.org/10.1080/00207546308947812

Huang, H. \& Irani, S.A. (2003, August 3-7). An enhanced systematic layout planning process for highvariety low-volume (HVLV) manufacturing facilities. Proceedings of the 17th International Conference on Production Research, Blacksburg, Virginia.

Irani, S. A., Zhang, H., Zhou, J., Huang, H., Udai, T. K. \& Subramanian, S. (2000). Production Flow Analysis and Simplification Toolkit (PFAST), International Journal of Production Research, 38(8), 1855-1874. https://doi.org/10.1080/002075400188636

Kahn, A. B. (1962). Topological sorting of large network. In: Communications of the ACM, 5(11), 558562.https://doi.org/10.1145/368996.369025

Kaparthi, S., Suresh, N.C. \& Cerveny, R.P. (1993). An improved neural network leader algorithm for partmachine grouping in group technology. European Journal of Operational Research, 69(3), 342-356. https://doi.org/10.1016/0377-2217(93)90020-N

King, J.R. (1980). Machine-component grouping in production flow analysis: an approach using a rank order clustering algorithm. International Journal of Production Research, $18(2), 213-232$. https://doi.org/10.1080/00207548008919662

Koren, Y., Hu J., \& Weber T. (1998). Impact of Manufacturing System Configuration on Performance. CIRP Annals, 47(1), 369-372. https://doi.org/10.1016/S0007-8506(07)62853-4

Kusiak, A. (1987). The generalized group technology concept. International Journal of Production Research, 25(4), 561-569. https://doi.org/10.1080/00207548708919861

Lee, D H., Kiritis, D., \& Xirouchakis, P. (2001). Search heuristics for operation sequencing in process planning. International Journal of Production Research, 39(16), 3771-3788. https://doi.org/10.1080/00207540110061922

Lee, D.H., Kiritsis, D., \& Xirouchakis, P. (2004). Iterative approach to operation selection and sequencing in process planning. International Journal of Production 
Research.

42

(22),

4745-4766.

https://doi.org/10.1080/00207540410001720412

Leone, G. \& Rahn, R.D (2002). Fundamentals of flow manufacturing. Flow publishing Inc. Boulder, Colorado, USA.

Lyonnet, B., \& Messaoudene, Z. (2012). Relations entre les pratiques de management et le système lean opérationnel d'entreprises françaises. Revue Française De Gestion Industrielle, 31(2), 63-82. https://doi.org/10.53102/2012.31.02.655

Mahesh, O. \& Srinivasan, G. (2002)., Incremental cell formation considering alternative machines. International Journal of Production Research, 40 (14), 3291-3310.

\section{https://doi.org/10.1080/00207540210146189}

Mayr, A., Weigelt, M., Kühl, A., Grimm, S., Erll, A., Potzel, M., \& Franke, J. (2018). Lean 4.0 - A conceptual conjunction of lean management and Industry 4.0. In Procedia CIRP, Volume 72 (pp. 622-628). Stockholm, Sweden. https://doi.org/10.1016/i.procir.2018.03.292

Muther, R. (1973). Systematic Layout Planning. 2nd Edition, Cahners Books, Boston.

Nicoletti B. (2013). Lean and Automate Manufacturing and Logistics. In: Prabhu V., Taisch M., Kiritsis D. (eds) Advances in Production Management Systems. Sustainable Production and Service Supply Chains. APMS 2013. IFIP Advances in Information and Communication Technology, vol 415. Springer, Berlin, Heidelberg. https://doi.org/10.1007/978-3-642-412639 34

Olhager, J. (2013). Evolution of Operations Planning and Control: From Production to Supply Chains. International Journal of Production Research, 51 (23-24), 6836-6843.

https://doi.org/10.1080/00207543.2012.761363

Phillips, E.J. (1997). Manufacturing Plant Layout: Fundamentals and Fine Points of Optimum Facility Design. Dearborn, MI: Society of Manufacturing Engineers.

Raoot, A.D. \& Rakshit, A. (1993). "A 'linguistic pattern' approach for multiple criteria facility layout problems", International Journal of Production Research, 31(1), 203222. https://doi.org/10.1080/00207549308956721

Real, R., Pralus, M., Pillet, M., \& Guizzi, L. (2010). Une première étape vers le Lean dans les entreprises de soustraitance mécanique, retour sur 7 ans de pratique. Revue Française De Gestion Industrielle, 29(1), 71-86. https://doi.org/10.53102/2010.29.01.619

Rouse, P. \& Putterill, M. (2003). An integral framework for performance measurement. Management
Decision, 41(8), 791-805. https://doi.org/10.1108/00251740310496305

Sarker, B.R. \& Xu, Y. (2000). " Designing multi-product lines: job routing in cellular manufacturing systems ", IIE Transactions. 32(3), 219-235. https://doi.org/10.1080/07408170008963894

Selim, H.M., Askin, R.G., Vakharia, A.J. (1998). "Cell formation in group technology: review, evaluation and directions for future research", Computers \& Industrial Engineering, 34(1), 3-20. https://doi.org/10.1016/S03608352(97)00147-2

Singh, S.P \& Sharma, R.R.K. (2006). A review of different aproaches to the facility layout problem, The International Journal of Advanced Manufacturing Technology, 30, 425-433. https://doi.org/10.1007/s00170-005-0087-9

Srinivasan, G., Narendran, T.T. \& Mahadevan, B. (1990). An assignment model for the part-families problem in Group Technology. International Journal of Production Research, 28(1), 145-152. https://doi.org/10.1080/00207549008942689

Tompkins, J. A., White, J. A., Bozer, Y. A., \& Tanchoco, J. M. A. (2010). Facilities planning. 4th Edition, John Wiley \& Sons.

Yin, Y., \& Yasuda, K. (2006). Similarity coefficient methods applied to the cell formation problem: $A$ taxonomy and review. International Journal of Production Economics, 101(2), 329-352. https://doi.org/10.1016/j.ijpe.2005.01.014

Zhou, J., \& Irani S. A. (2003). New flow diagramming scheme for mapping and analysis of multi-product flows in facility. Journal of Integrated Design and Process Science, $\quad 7(1), \quad 25-58$. https://content.iospress.com/articles/journal-ofintegrated-design-and-process-science/jid7-1-03

Zhou J., Barth, M. \& De Guio R. (1996). Quasi-source heuristic for the production line formation of manufacturing system. IEEE International Conference on Systems, Man and Cybernetics. Information Intelligence and Systems (Cat. No.96CH35929), 4, pp. 3009-3014. https://doi.org/10.1109/ICSMC.1996.561444.

\section{BIOGRAPHIE}

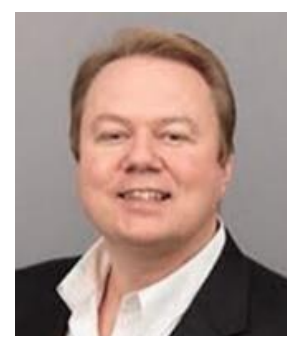

David Damand : est maître de conférences $(\mathrm{HC})$ à I'EM Strasbourg (Université de Strasbourg, France) et chercheur au laboratoire HUMANIS. II enseigne la planification de la chaîne 
logistique. Ses travaux portent sur l'analyse décisionnelle multicritères pour la planification de la chaîne logistique et la méthodologie de conception d'entrepôts.

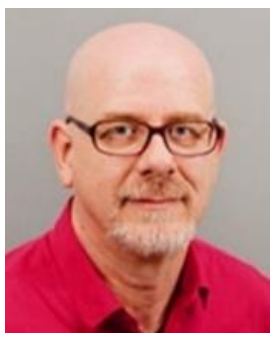

Marc Barth : est maître de conférences habilité à diriger les recherches en génie industriel à I'INSA de Strasbourg en France. II est membre du laboratoire de recherche HUMANIS de I'EM

Strasbourg. Ses recherches et son enseignement portent sur les applications de la planification et du contrôle de la production, la théorie de la résolution de problèmes inventifs, l'application des méthodes activity based costing et time driven activity based costing et la conception des systèmes de production et des entrepôts.

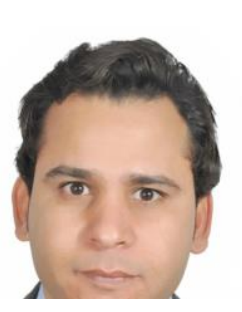

Abdehlatif Dhkil : est professeur associé en génie industriel à l'Ecole de Technologie Supérieur Département de génie de la production automatisée (Université du Québec à
Montreal, Canada) et chercheur au LIPPS (Laboratoire d'Ingénierie des Produits, Procédés et Systèmes). Ses recherches portent sur la conception de systèmes de production et la représentation de carte cognitives pour l'analyse.

${ }^{1}$ David Damand, HuManis laboratory of EM Strasbourg Business School, 61 avenue de la Forêt Noire, F-67000, Strasbourg, France, damand@unistra.fr,

\section{(1) https://orcid.org/0000-0002-6187-2115}

${ }^{2}$ Marc Barth, HuManis laboratory of EM Strasbourg Business School, 61 avenue de la Forêt Noire, F67000, Strasbourg, France, marc.barth@insastrasbourg.fr,

https://orcid.org/0000-0001-6752-4384

${ }^{3}$ Abdellatif Dhkil, Université du Québec, Ecole de Technologie Supérieure, LIPPS, 1100 rue Notre Dame Ouest, Montréal, H3C 1K3, Canada, abdellatif.dkhil@gmail.com 